\title{
Inferior Phrenic Arteries Originating from the Celiac Artery with a Common Trunk
}

\section{Ortak Bir Kütükle Truncus Coeliacus'tan Çıkan Sağ ve Sol Arteria Phrenica Inferior}

Yasin ARIFOGLU1', Mehmet YILDIRIM², Huseyin BALCIOGLU³

${ }^{1}$ Department of Anatomy, Faculty of Medicine, Bezmialem Vakif University, Istanbul, Turkey

${ }^{2}$ Department of Anatomy, Cerrahpasa Faculty of Medicine, Istanbul University, Istanbul, Turkey

${ }^{3}$ Department of Anatomy, Faculty of Dentistry, Istanbul University, Istanbul, Turkey

\section{ABSTRACT}

The inferior phrenica artery (IPA), which arises from the abdominal aorta just distal to the aortic hiatus, may arise from different sites. Although comprehensive analyses of the IPA are described in other populations, the rates of the variations of the origins are not well elucidated in Turkish people. In this study, we report IPA arising as a common trunk from the abdominal aorta. This finding in a 52 year old male cadaver was noticed during a routine dissection for students in the 2010-2011 academic year. We believe this report is a valuable contribution to the literature on variation of the phrenic arteries and is of interest to clinicans in the surgical and interventional radiological procedures of the adrenal glands, spleen, liver and stomach as well as to the educators of anatomy.

Key Words: Abdominal aorta, celiac artery, inferior phrenic artery, variation.

\section{ÖZET}

Genel olarak hiatus aorticus'un hemen aşağısında aorta abdominalis'ten çıkan arteria phrenic inferior'lar (IPA) sağda ve solda farklı yerlerden de çıkabilirler. Bu konuda ülkemiz dışında yapılan geniş serili çalışmalar klasik kitaplara geçen bilgiler haline gelmesine karşın Türkiye'de yaşayan insanlarda IPA'ların farklı çıkış yeri oranları tam olarak çıkarılmamıştır. 2010-2011 öğretim yılında, öğrenci eğitim kadavralarının diseksiyonları esnasında 52 yaşında bir erkek kadavrada, IPA’ların ortak bir kütükle truncus coelicus'tan çıktığı görüldü. Böbrek üstü bezleri, dalak, karaciğer ve mide cerrahisi esnasında ve bölgenin girişimsel radyolojik prosedürlerinde olduğu kadar, anatomi öğretiminde de önem taşıyan bu frenik arter varyasyonunun, klinisyenler ve anatomistler için literatüre bir katkı sağlayacağını düşünmekteyiz.

Anahtar Sözcükler: Aorta abdominalis, truncus coeliacus, arteria phrenica inferior, varyasyon

\section{Giriş}

Truncus coeliacus'un hemen yukarısında olarak aorta abdominalis'in ön yüzünden sağlı sollu çıkan iki arteria phrenica inferior (IPA), diaphragma’nın crus'larını çaprazlayarak dış yana doğru uzanırlar. Sol IPA, oesophagus'un arkasından geçip hiatus oesophagus'un sol kenarı boyunca ilerler. Sağ IPA ise vena cava inferior'un arkasından geçerek foramen vena cava’nın sağ kenarı boyunca uzanır. Centrum tendineum’un arka bölümüne yakın olarak her iki arter medial ve lateral dallarına ayrılır. Medial dal öne doğru kıvrılarak karşı tarafın aynı dalı, a. musculophrenica ve a. pericardiacophrenica ile anastomoz yapar. Lateral dal ise toraks duvarına doğru uzanarak alt interkostal arterler ve a. musculophrenica ile anastomoz yapar. IPA'lardan, oesophagus, suprarenal bez, dalak ve karaciğer'e giden dallar çıkar (1-3). IPA'lar aorta, truncus coeliacus, sol gastrik ve hepatik arterden, a. renalis, a. testicularis (a. ovarica) olmak üzere değişik yerlerden çıabilir (4) .

\section{Olgu Sunumu}

2010-2011 öğretim yılında, öğrenci eğitim kadavralarının karın arka duvarı diseksiyonlarında 52 yaşında bir erkek kadavranın, aorta abdominalis'inin dallarının ortaya konması esnasında, IPA’ların aorta abdominalis'ten kaynaklanmadığı görüldü. Olası varyasyonel çıkış yerlerinden biri olan truncus coeliacus dikkatlice ortaya konduğunda, çıkışının $7 \mathrm{~mm}$ distalinin üst yüzünden IPA’ların ortak bir kütükle çıktığı görüldü. Varyasyonel olgunun resmi çekildi ve kumpas ile öl- 
çümleri yapıldı. Ortak kütügün çapı 3,6 mm, kütükten $1 \mathrm{~cm}$ distalde sağ a.phrenica inferior $3 \mathrm{~mm}$, sol a.phrenica inferior yine $3 \mathrm{~mm}$ olarak ölçüldü (Resim 1).

\section{Tartı̧̧ma}

Prensip dallanma şekline göre aorta abdominalis'ten ayrı ayrı çıkan sağ-sol IPA, belli oranlarda ortak kütük halinde veya ayrı ayrı farklı yerlerden de orijin alabilir (3-7). Clemente (3) olguların üçte birinde IPA'ların ortak bir kütük halinde aorta abdominalis veya truncus coeliacus'tan çıktığını belirtmektedir.

Lippert ve Pabst (4) ve Michels (5) IPA'ları ortak kütükle veya ayrı ayrı çıkma durumuna göre iki gruba ayırırken Anson ve McVay (6) arteri orijinlerine göre yedi grupta toplamıştır. Ortak kütükle çıkma oranı Lippert ve Pabst'a (4) göre \%33, Michels'e (5) göre \%40'tır.

Ortak kütükle çıktıkları durumlarda arter, aorta abdominalis, truncus coeliacus veya a. gastrica sinistra'dan orijin alabilmektedir. Bu tiple ilgili oranlar Lippert ve Pabst'a göre (4) sırasıla $\% 18, \% 14, \% 1$ 'dir. Çavdar ve ark. (7), sol inferior frenik arter ve sol gastrik arterin uzun ortak bir kütük şeklinde $(4,3 \mathrm{~cm})$ truncus coeliacus'tan çıktığını rapor etmişlerdir. Songur ve ark. (8) yaptıkları çalışmada IPA'ların truncus coeliacus'tan tek kök olarak çıktığı olguların oranını \%4,2 olarak bildirmiştir.

Arteria phrenica inferior'ların ayrı ayrı çıkma oranı Lippert ve Pabst'a (4) göre \%67, Michels'e (5) göre \%60'tır. IPA’ların ayrı ayrı çıtıkları durumda orijin yerleri aorta abdominalis, truncus coeliacus, a. gastrica sinistra, a. hepatica propria veya a.testicularis (a. ovarica) olabilmektedir $(4,5)$. A. hepatica propria ve a. testicularis (a. ovarica)'den çıkış oranı çok düşük olarak belirtilmektedir. Anson ve McVay'e (6) göre aorta abdominalis'den orijin alma oranı \%31, truncus coeliacus'tan $\% 38$, sağ a. renalis'ten $\% 7^{\prime}$ dir.

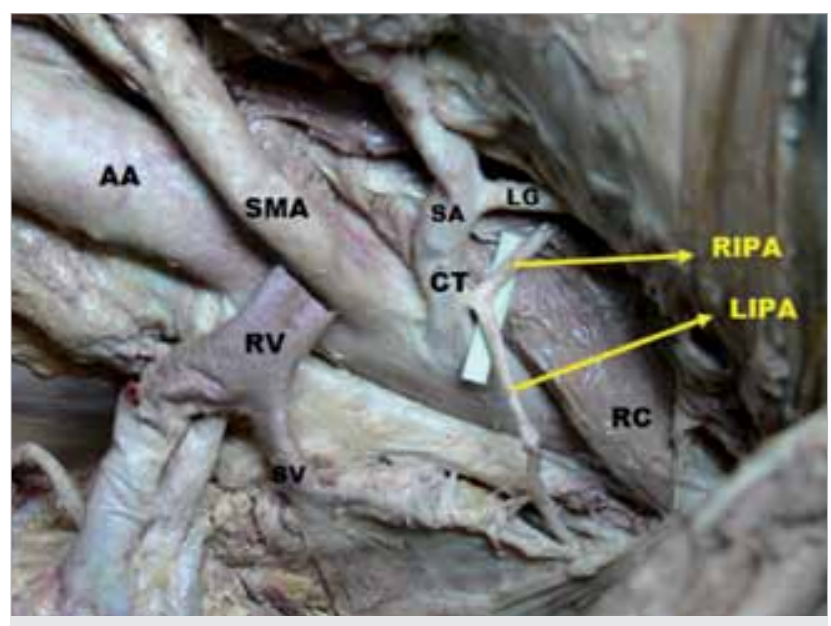

Resim 1. Ortak bir kütükle truncus coeliacus'tan çıkan sağ ve sol a. phrenica inferior'lar

RIPA: A. phrenica inferior dextra; LIPA: A. phrenica inferior sinistra; RV: V. renalis sinistra; SMA: A. mesenterica superior; AA: Aorta abdominalis; CT: Truncus coeliacus; SV: V. suprarenalis sinistra; RC: Crus dextrum; LG: $A$. gastrica sinistra; SA: $A$. splenica
Karaciğer diafragma ve IPA'nın dalları ile temas halindedir. IPA'nın, hepatosellüler karsinomların ekstrahepatik kollateral beslenmesinde major kaynak olması nedeniyle, hepatosellüler karsinomların tedavisinde transarteriel kemoembolizasyon uygulamalarında IPA'nın varyasyonel anatomi bilgisi tanı, tedavi ve kateterizasyon teknikleri açısından hayati önem arzetmektedir (9). Bu türden örnekler birçok cerrahi ve girişimsel radyoloji uygulamaları için çoğaltılabilir.

\section{Sonuç}

Arteria phrenica inferior'un ortak bir kütük ile truncus coeliacus'tan çıkış varyasyonu olarak sunduğumuz bu vakanın, klinisyenler için söz konusu arterin varyasyonel bilgisini gözden geçirmesi açısından literatüre bir katkı olacağını düşünmekteyiz.

Conflict of Interest: No conflict of interest was declared by the authors.

Peer-review: Externally peer-reviewed.

Author Contributions: Concept - Y.A., M.Y., H.B.; Design Y.A., M.Y., H.B.; Supervision - Y.A., M.Y., H.B.; Funding - Y.A., M.Y., H.B.; Materials - Y.A., M.Y., H.B.; Data Collection and/or Processing - Y.A., M.Y., H.B.; Analysis and/or Interpretation - Y.A., M.Y., H.B.; Literature Review - Y.A., M.Y., H.B.; Writing - Y.A., M.Y., H.B.; Critical Review - Y.A., M.Y., H.B.; Other - Y.A., M.Y., H.B.

Çıkar Çatışması: Yazarlar çıkar çatışması bildirmemişlerdir.

Hakem değerlendirmesi: Dış bağımsız.

Yazar Katkıları: Fikir - Y.A., M.Y., H.B.; Tasarım - Y.A., M.Y., H.B.; Denetleme - Y.A., M.Y., H.B.; Kaynaklar - Y.A., M.Y., H.B.; Malzemeler - Y.A., M.Y., H.B.; Veri toplanması ve/veya işlemesi - Y.A., M.Y., H.B.; Analiz ve/veya yorum - Y.A., M.Y., H.B.; Literatür taraması - Y.A., M.Y., H.B.; Yazıyı yazan - Y.A., M.Y., H.B.; Eleştirel İnceleme - Y.A., M.Y., H.B.; Diğer - Y.A., M.Y., H.B.

\section{Kaynaklar}

1. Williams PL, Bannister LM, Berry MM. Gray's Anatomy, $38^{\text {th }}$ ed, Churchill and Livingstone, London; 1995.p.1558.

2. Moore KL, Dalley AF. Clinically Oriented Anatomy, $4^{\text {th }}$ Edition, Lippincott Williams \&Wilkins, Philadelphia, New York; 1999.p.280-9.

3. Clemente CD. Gray's Anatomy, Thirteenth American Edition LEA and Febiger Philadelphia; 1985.p.746-7.

4. Lippert H, Pabst R. Arterial Variations in Man, JF Bergmann Verlag München; 1985.p.24. [CrossRef]

5. Michels NA. Blood Supply and Anatomy of the Upper Abdominal Organs with a Descriptive Atlas Lippincott, Philadelphia; 1955.p.128.

6. Anson B, Mc Way CB. Surgical anatomy, Vol. $15^{\text {th }}$ ed. W B Saunder Company, Philadelphia; 1971.p.372.

7. Cavdar S, Gürbüz J, Zeybek A, Sehirli U, Abik L, Ozdogmuş O: A variation of coeliac trunk. Kaibogaku Zasshi 1998; 73: 505-8.

8. Ahmet Songur et al. Abdominal Aorta and Its Branches: Morphometry - Variations In Autopsy Cases. Eur J Gen Med 2010; 7: 321-5.

9. Miyayama S, Yamashiro M, Yoshie Y, Okuda M, Nakashima Y, Ikeno $\mathrm{H}$, et al. Inferior phrenic arteries: angiographic anatomy, variations, and catheterization techniques for transcatheter arterial chemoembolization. Jpn J Radiol 2010; 28: 502-11. [CrossRef] 